\title{
QCD with Chiral Imbalance: models vs. lattice
}

\author{
Alexander Andrianov ${ }^{1,2, a}$, Vladimir Andrianov ${ }^{1, b}$, and Domenec Espriu ${ }^{2, c}$ \\ ${ }^{1}$ Department of Physics, Saint Petersburg State University, Universitetskaya nab. 7/9, Saint Petersburg \\ 199034, Russia, \\ ${ }^{2}$ Departament de Física Quàntica i Astrofísica and Institut de Ciéncies del Cosmos (ICCUB), Universitat de \\ Barcelona, Martí i Franqués 1, 08028 Barcelona, Spain
}

\begin{abstract}
In heavy ion collisions (HIC) at high energies there may appear new phases of matter which must be described by QCD. These phases may have different color and flavour symmetries associated with the constituents involved in collisions as well as various space-time symmetries of hadron matter. Properties of the QCD medium in such a matter can be approximately described, in particular, by a number of right-handed $(\mathrm{RH})$ and left-handed (LH) light quarks. The chiral imbalance (ChI) is characterized by the difference between the numbers of RH and LH quarks and supposedly occurs in the fireball after HIC. Accordingly we have to introduce a quark chiral (axial) chemical potential which simulates a $\mathrm{ChI}$ emerging in such a phase. In this report we discuss the possibility of a phase with Local spatial Parity Breaking (LPB) in such an environment and outline conceivable signatures for the registration of LPB as well as the appearance of new states in the spectra of scalar, pseudoscalar and vector particles as a consequence of local ChI. The comparison of the results obtained in the effective QCD- motivated models with lattice data is also performed.
\end{abstract}

\section{Introduction and Outline}

The formation of phase with the Chirality imbalance (ChI) i.e. with a difference between average numbers of right-handed (RH) and left-handed (LH) quarks in the fireball after heavy-ion collisions (HIC) represents an interesting challenge in studies of the phase diagram of QCD. Among several reasons to unravel this phenomenon there is a nontrivial topology of QCD vacuum [1-5], the famous chiral Adler-Bell-Jackiw anomaly [6, 7], an appearance in non-central HIC of so called Chiral Magnetic Effect (CME) and other $C$ - and $C P$ - violating processes [8], the problem of determination of the location of the Critical End Point (CEP) of QCD [9]. Finally the lattice simulations of QCD with chiral (axial) chemical potential [10] may shed light on matter with $\mathrm{ChI}$ and can be further on exploited as a basis for comparing different effective models with $\mathrm{ChI}$ used in the literature.

In order to describe a matter with $\mathrm{ChI}$ a chiral (axial) chemical potential can be introduced to quantify the influence of chirality imbalance. On the other hand the process of creating nonzero chirality pertains to the famous chiral ABJ-anomaly $[6,7]$. This anomaly implies the breaking of

\footnotetext{
a e-mail: andrianov@icc.ub.edu

be-mail: v.andriano@rambler.ru

ce-mail: espriu@icc.ub.edu
} 
axial current conservation by quantum loop effects. Correspondingly, locally, chirality imbalance can be generated through nonorthogonal electric-like and magnetic-like field strengths. Such fields can be either QED EM fields or QCD chromo-EM ones. In QCD typical gluon configurations generating ChI exist: they are the topological solitons such as instantons and sphalerons. Global configurations of this type have their global topological winding number $Q_{w}$, and when coupled with chiral fermions, can generate an amount of global chirality imbalance via chiral anomaly: $N_{R}-N_{L}=2 Q_{w}$ for each flavor of light quarks. The quark masses and a nonzero quark condensate mix right- and left-handed quarks and have the effect of decreasing the chiral asymmetry. Therefore, as one enforces a system to generate the right-left asymmetry by increasing chiral chemical potential, one would expect that quark condensates tended to become larger and thereby critical temperature $T_{c}$ of chiral symmetry restoration were to rise as well. It happens that chirality imbalance is a direct manifestation of QCD topological fluctuations and could become observable through the asymmetry in polarizations of dilepton pairs [11] in central HIC as a result of the manifestation of Local spatial Parity Breaking (LPB) effect.

In the talk the phenomenology of formation of Local spatial Parity Breaking (LPB) in the matter with ChI is discussed, its description within a number of QCD-inspired models is outlined and put in comparison with lattice QCD simulation.

We focus on and consider:

- where chiral imbalance appears - phenomenology of parity breaking in finite volume;

- topological charge and axial (chiral) charge in fireball $\Rightarrow$ topological axial (chiral) chemical potentials;

- phenomenology of chiral imbalance in non-Abelian quark-gluon phase;

- chiral imbalance in hadronic phase - influence on chiral symmetry breaking (on chiral condensates);

- observables sensitive to the local chiral imbalance - manifestation of LPB in central HIC: dilepton polarization asymmetry [11]

- new states in scalar, pseudoscalar, vector meson spectra in hadrom matter with ChI.

\section{Phenomenology of local parity breaking in finite volume}

Global spatial parity symmetry is a well established global symmetry of strong interactions. But in a hot and dense matter of finite-volume fireball local fluctuations in the topological charge apparently exist and they may be responsible for the generation of LPB. In particular:

- Quantum fluctuations of $\theta$ parameter: $P$-odd bubbles [12], their manifestation in Chiral Magnetic Effect (CME)[13]) (an earlier proposal: [14] ).

- New QCD phase characterized by a spontaneous parity breaking due to formation of neutral pionlike background $[15,16]$.

- High energy production of pseudoscalar gluelumps $\Leftrightarrow$ of parity-odd bunches of gluon jets $\Rightarrow$ then a PB background remains inside a hot dense nuclear fireball in HIC !?

Observable effects:

- For peripheral heavy ion collisions they lead to the Chiral Magnetic Effect (CME): Large $\vec{B} \Rightarrow$ large $\vec{E} \Rightarrow$ charge separation[8] (and the D.Kharzeev's talk on this conference).

- For central collisions (and light quarks) they correspond to a phase with axial chemical potential $\mu_{5} \neq 0$ located in "fluctons" of few-Fermi size [11].

These two effects are complementary and coexist in HIC! 


\section{Topological charge and topological chemical potential}

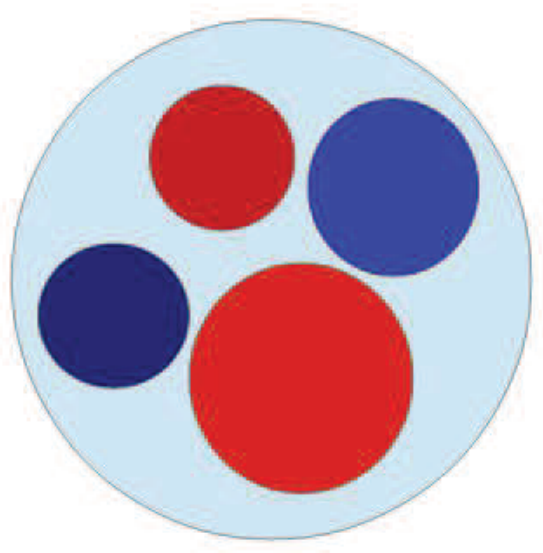

Figure 1. Interplay between domains with positive (in red) and negative (in blue) topological charge in the fireball interior

In hot QCD (in heavy ion collisions) a metastable topological charge $\left\langle T_{5}\right\rangle$ may arise in the limit of infinite volume due to sphaleron-like transitions [17]. However strictly speaking in a finite volume and for a fixed time there must be some other mechanisms only qualitatively associated with sphalerons but without generation of quark zero modes. Their role is to provide a nontrivial metastable topological charge in a compact space-time region:

$$
T_{5}=\frac{1}{8 \pi^{2}} \int_{\text {vol. }} d^{3} x \varepsilon_{j k l} \operatorname{Tr}\left(G^{j} \partial^{k} G^{l}-i \frac{2}{3} G^{j} G^{k} G^{l}\right) .
$$

It must survive for a sizeable lifetime in a heavy-ion fireball,

$$
\left\langle\Delta T_{5}\right\rangle \neq 0 \text { for } \quad \Delta t \simeq \tau_{\text {fireball }} \simeq 5 \div 10 \mathrm{fm} / \mathrm{c}
$$

$$
(7 \pm 1 \mathrm{fm} / \mathrm{c}[18]) \text {, }
$$

with relatively negligible topological current fluxes acroww the boundary.

For the fireball lifetime one can trigger the value of $\left\langle\Delta T_{5}\right\rangle \neq 0$ introducing into the QCD Lagrangian a topological chemical potential $\mu_{\theta}$ in a gauge invariant way via $\Delta \mathcal{L}_{\text {top }}=\mu_{\theta} \Delta T_{5}$, where

$$
\Delta T_{5}=T_{5}\left(t_{f}\right)-T_{5}(0)=\frac{1}{8 \pi^{2}} \int_{0}^{t_{f}} d t \int_{\text {vol. }} d^{3} x \operatorname{Tr}\left(G^{\mu \nu} \widetilde{G}_{\mu v}\right) .
$$

We notice that in a finite $3+1$ dimensional volume the so-called index theorem does not work and the jump of topological charge is not necessarily integer.

\section{Axial baryon charge and axial chemical potential}

The exact operator law in QCD, the partial conservation of axial current (broken by current quark masses $m_{q}$ for light quarks $q=u, d, s$ and gluon anomaly)

$$
\partial_{\mu} J_{5}^{\mu}-2 i m_{q} J_{5}=\frac{1}{8 \pi^{2}} \operatorname{Tr}\left(G^{\mu v} \widetilde{G}_{\mu \nu}\right)
$$


predicts the rigid relations between changes in topology of gauge configurations and the induced axial charge (for small quark masses $m_{q} \simeq 0$ ). If the gluon and quark flows across the volume boundary of a fireball are reasonably small (a fireball is metastable) then,

$$
\frac{d}{d t}\left(Q_{5}-2 N_{f} T_{5}\right) \simeq 0, \quad Q_{5}=\int_{\text {vol. }} d^{3} x \sum_{u, d, s} q^{\dagger} \gamma_{5} q=\left\langle N_{L}-N_{R}\right\rangle,
$$

and the chiral charge generated in a heavy ion collision is approximately conserved during $\tau_{\text {fireball }}$.

The characteristic left-right oscillation time is governed by inverse quark masses. Indeed if an excess of left(or right)-handed quark states $q_{L}\left(x_{0}=0\right)$ arises in the fireball in the initial moment of central collision then their further evolution in the rest frame can be approximately characterized by the following equation,

$$
q\left(x_{0}\right) \simeq\left(\cos \left(m_{q} x_{0}\right)-i \gamma_{0} \sin \left(m_{q} x_{0}\right)\right) q_{L}(0) .
$$

Therefore after a period $\Delta x_{0} \sim \pi / 2 m_{q}$ left-handed quark states become essentially right-handed.

- For $u, d$ quarks $1 / m_{q} \sim 1 / 5 \mathrm{MeV}^{-1} \sim 40 \mathrm{fm} \gg \tau_{\text {fireball }}$ and the left-right quark mixing can be neglected during fireball life time.

- For $s$ quark $1 / m_{s} \sim 1 / 150 \mathrm{MeV}^{-1} \sim 1 \mathrm{fm} \ll \tau_{\text {fireball }}$ and the chiral charge averaged over the fireball volume and its lifetime vanishes $\left\langle Q_{5}^{s}\right\rangle \simeq 0$ due to left-right oscillations.

Correspondingly for $u, d$ quarks in a background with the jump of topological charge $\left\langle\Delta T_{5}\right\rangle \neq 0$ or with the chiral (axial) charge $\left\langle Q_{5}\right\rangle \neq 0$ QCD can be equally described at the Lagrangian level by introducing a topological chemical potential $\mu_{\theta}$ or an axial chemical potential $\mu_{5}$

$$
\begin{gathered}
\left\langle\Delta T_{5}\right\rangle \simeq \frac{1}{2 N_{f}}\left\langle Q_{5}\right\rangle \Longleftrightarrow \mu_{5} \simeq \frac{1}{2 N_{f}} \mu_{\theta}, \\
\Delta \mathcal{L}_{\text {top }}=\mu_{\theta} \Delta T_{5} \Longleftrightarrow \Delta \mathcal{L}_{q}=\mu_{5} Q_{5}
\end{gathered}
$$

Thus one can think about a generation of chiral imbalance from non-trivial topological charge or visa versa inducing a topologically non-trivial gluon configuration by imbalance of left- and right-handed quark densities. As both properties are related locally one may get a parity violation generated by either charge in a finite volume of fireball and visible during fireball lifetime.

How does $\mu_{5}$ affect the hadronic phenomenology?

\section{Effective meson theory in a medium with LPB}

Thus in the environment with chiral chemical potential the QCD quark lagrangian obtains the term $\Delta \mathcal{L}_{q}=\mu_{5} \bar{q} \gamma_{0} \gamma_{5} \mathbf{I}_{q} q$, i.e. the time-like component of axial-vector current containing $u, d$ quarks only. After bosonization of QCD the scalar sector can be described by using the spurion technique in the chiral Lagrangian with an external isosinglet axial-vector current $A_{v}=\mu_{5} \delta_{0 v}$ [19], $D_{v} \Longrightarrow D_{v}-i\left\{\mathbf{I}_{q} \mu_{5} \delta_{0 v}, \star\right\}$, where for three light flavors $\mathbf{I}_{q} \equiv \operatorname{diag}[1,1,0]$ in accordance with the preceding discussion. An effective Lagrangian is required to include the lightest scalar degrees of freedom such as $\sigma$ and $a_{0}(980)$, which are mixed with their pseudoscalar partners $\eta, \eta^{\prime}$ and $\pi$, respectively.

In the lowest order of chiral expansion such an effective lagrangian reads,

$$
\mathcal{L}=\frac{1}{4} \operatorname{Tr}\left(D_{\mu} H D^{\mu} H^{\dagger}\right)+\frac{B}{2} \operatorname{Tr}\left[\hat{m}\left(H+H^{\dagger}\right)\right]+\frac{M^{2}}{2} \operatorname{Tr}\left(H H^{\dagger}\right)
$$




$$
-\frac{\lambda_{1}}{2} \operatorname{Tr}\left[\left(H H^{\dagger}\right)^{2}\right]-\frac{\lambda_{2}}{4}\left[\operatorname{Tr}\left(H H^{\dagger}\right)\right]^{2}+\frac{c}{2}\left(\operatorname{det} H+\operatorname{det} H^{\dagger}\right)
$$

where the $S U(3)_{L} \times S U(3)_{R}$ chirally invariant part is parameterized by constants $M, \lambda_{1,2}, c$ and soft chiral symmetry breaking is realized with the help of the vertex containing $\hat{m}=\operatorname{diag}\left[m_{u}, m_{d}, m_{s}\right]$. The convenient splitting of scalar fields is given by

$$
H=\xi \Sigma \xi, \quad \xi=\exp \left(i \frac{\Phi}{2 f_{\pi}}\right), \quad \Phi=\lambda^{a} \phi^{a}, \quad \Sigma=\lambda^{b} \sigma^{b} .
$$

We include the non-strange meson sector and $\eta_{s}$

$$
\begin{gathered}
\Phi=\left(\begin{array}{ccc}
\eta_{q}+\pi^{0} & \sqrt{2} \pi^{+} & 0 \\
\sqrt{2} \pi^{-} & \eta_{q}-\pi^{0} & 0 \\
0 & 0 & \sqrt{2} \eta_{s}
\end{array}\right), \Sigma=\left(\begin{array}{ccc}
v_{u}+\sigma+a_{0}^{0} & \sqrt{2} a_{0}^{+} & 0 \\
\sqrt{2} a_{0}^{-} & v_{d}+\sigma-a_{0}^{0} & 0 \\
0 & 0 & v_{s}
\end{array}\right), \\
\left(\begin{array}{l}
\eta_{q} \\
\eta_{s}
\end{array}\right)=\left(\begin{array}{cc}
\cos \psi & \sin \psi \\
-\sin \psi & \cos \psi
\end{array}\right)\left(\begin{array}{l}
\eta \\
\eta^{\prime}
\end{array}\right) .
\end{gathered}
$$

For $\mu_{5}=0$, in the chiral limit $\hat{m}=0$ we adopt $v_{u}=v_{d}=v_{s}=v_{0} \equiv f_{\pi} \approx 92 \mathrm{MeV}$ where $v_{i}=\left\langle\Sigma_{i i}\right\rangle$. The coupling constants (in units of $\mathrm{MeV}$ ) are fitted to phenomenology assuming isospin symmetry. The v.e.v. of the neutral scalars, in the chiral limit $\hat{m}=0$, satisfy the following gap equations:

$$
\begin{gathered}
\left(2 \mu_{5}^{2}+M^{2}\right)-2\left(\lambda_{1}+\lambda_{2}\right) v_{q}^{2}-\lambda_{2} v_{s}^{2}+c v_{s}=0, \\
\left(-2 \lambda_{2}+\frac{c}{v_{s}}\right) v_{q}^{2}+M^{2}-\left(2 \lambda_{1}+\lambda_{2}\right) v_{s}^{2}=0,
\end{gathered}
$$

where for non-vanishing isosinglet $\mu_{5}$ we find our solutions to be $v_{u}=v_{d} \equiv v_{q} \neq v_{s}$.

Evidently for large $\mu_{5}$ the correct solution has the following asymptotics,

$$
v_{q}^{2}=\frac{2 \mu_{5}^{2}+M^{2}+\frac{c^{2}}{4 \lambda_{2}^{2}}}{2\left(\lambda_{1}+\lambda_{2}\right)}+O\left(1 / \mu_{5}^{2}\right), \quad v_{s}=\frac{c}{2 \lambda_{2}}+O\left(1 / \mu_{5}^{2}\right) .
$$

These solutions are depicted in the Fig.2.

We stress that the consistent solution with a finite limit for strange quark constituent mass $v_{s} \ll v_{q}$ exists if the term breaking $U(1)_{A}$ symmetry is in action. Meantime the constituent masses for light $u, d$ flavours is growing with chiral chemical potential, first as $\sim \mu_{5}^{2}$ and next as $\sim \mu_{5}$. Moreover even if the spontaneous chiral symmetry breaking is absent for $M^{2}<0$ in parity-even environment this CSB will be triggered by chiral imbalance. The lattice computations [10] shown on Fig.3 confirm this behavior against chiral chemical potential.

We notice that the condensates can be derived from the effective lagrangians by variation in current quark masses and therefore $-\langle\bar{\psi} \psi\rangle_{q}=B v_{q}\left(\mu_{5}\right)$ which allows us to compare the chiral imbalance in effective lagrangian models[19] and in lattice simulations [10], as it is shown in Figs.2 and 3. We see that the estimations for condensate dependence on chiral chemical potential done in the effective lagrangian approach are perfectly confirmed by lattice computations. Therefore one can exploit the meson effective lagrangians for predictions of mass spectrum and decay constants of lightest mesons in the chiral imbalanced environment. 


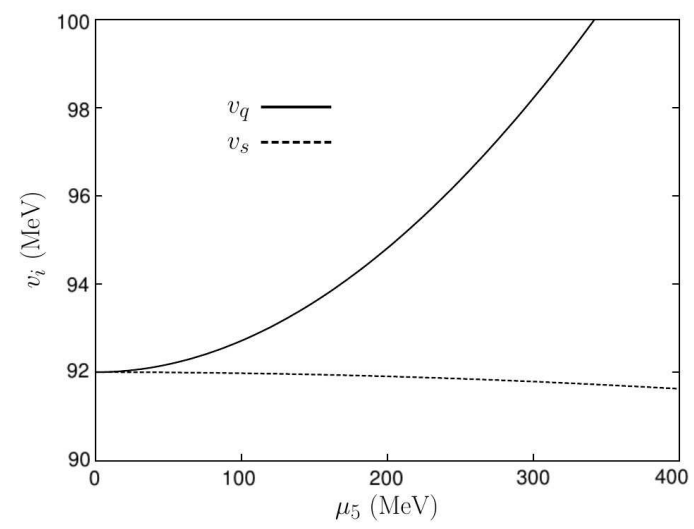

Figure 2. Vacuum condensates in the presence of chiral imbalance

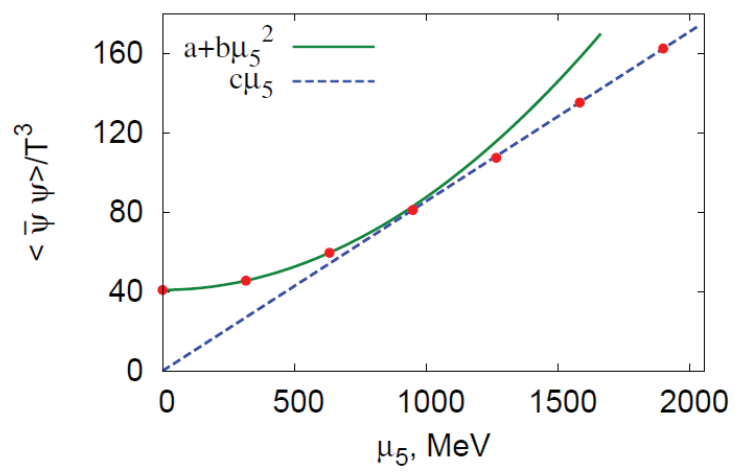

Figure 3. Chiral condensate in two-color QCD lattice as a function of $\mu_{5}$ in the confinement phase $(\mathrm{T}=158$ $\mathrm{MeV})$.

\subsection{New eigenstates of strong interactions with LPB (isotriplet)}

Following to [19] we present a simple case of mixing due to LPB in the isotriplet sector with $\pi$ and $a_{0}$. The kinetic and mixing terms in the Lagrangian are given by

$$
\mathcal{L}=\frac{1}{2}\left(\partial a_{0}\right)^{2}+\frac{1}{2}(\partial \pi)^{2}-\frac{1}{2} m_{1}^{2} a_{0}^{2}-\frac{1}{2} m_{2}^{2} \pi^{2}-4 \mu_{5} a_{0} \dot{\pi}
$$

where

$$
\begin{aligned}
& m_{1}^{2}=-2\left[M^{2}-2\left(3 \lambda_{1}+\lambda_{2}\right) v_{q}^{2}-\lambda_{2} v_{s}^{2}-c v_{s}+2 \mu_{5}^{2}\right] \\
& m_{2}^{2}=\frac{2 m}{v_{q}} B .
\end{aligned}
$$

After diagonalization in the momentum representation, the new (momentum-dependent) eigenstates are defined $\tilde{\pi}$ and $\tilde{a}_{0}$.

$\tilde{a}_{0}$ mass shows an enhancement, but $\mu_{5}$ has to be understood as a perturbatively small parameter. A better treatment of $\tilde{a}_{0}$ would require heavier degrees of freedom. 

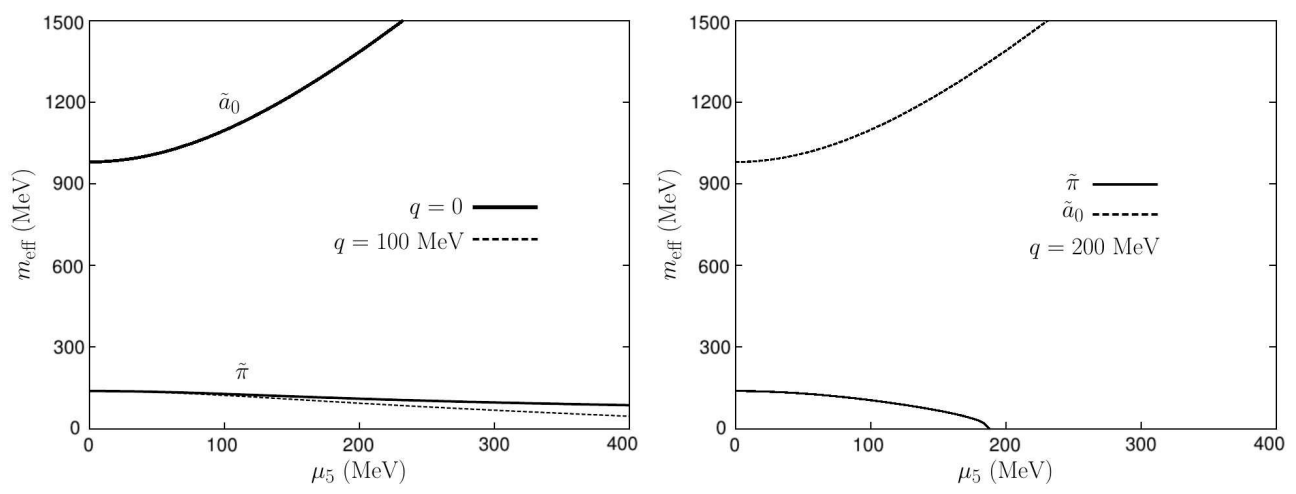

Figure 4. For high energies $k_{0},|\vec{k}|>m_{1} m_{2} /\left(4 \mu_{5}\right) \equiv k_{\tilde{\pi}}^{c}$, in-medium $\tilde{\pi}$ goes tachyonic. Nevertheless, energies are always positive (no vacuum instabilities).

\subsection{New eigenstates of strong interactions with LPB (isosinglet)}

Two new processes are likely to appear inside the fireball: the decays $\eta, \eta^{\prime} \rightarrow \pi \pi$ that are strictly forbidden in QCD on parity grounds [19].

In a medium where parity is broken: are these processes relevant within the fireball? Can these particles reach thermal equilibrium?!

There are no $O\left(p^{2}\right)$ terms in the chiral Lagrangian involving vertices $\eta \pi \pi$.

$O\left(p^{4}\right)$ terms lead to couplings such as

$$
\mathcal{L}_{\eta \pi \pi} \sim \frac{16 \mu_{5}}{F_{\eta} f_{\pi}^{2}} L \partial \eta \partial \pi \partial \pi,
$$

where $L \sim 10^{-3}$. A rough estimate of the partial width shows a strong dependence on $\mu_{5}$ as $\Gamma_{\eta \rightarrow \pi \pi} \propto \mu_{5}^{2}$ and gives values similar or higher than $\Gamma_{\rho \rightarrow \pi \pi}=150 \mathrm{MeV}$.

In the isosinglet sector, we show the mixing of $\eta, \sigma$ and $\eta^{\prime}$. The kinetic and mixing terms in the Lagrangian are given by [19]

$$
\begin{aligned}
\mathcal{L}= & \frac{1}{2}\left[(\partial \sigma)^{2}+\left(\partial \eta_{q}\right)^{2}+\left(\partial \eta_{s}\right)^{2}\right]-\frac{1}{2} m_{3}^{2} \sigma^{2}-\frac{1}{2} m_{4}^{2} \eta_{q}^{2}-\frac{1}{2} m_{5}^{2} \eta_{s}^{2} \\
& -4 \mu_{5} \sigma \dot{\eta}_{q}-2 \sqrt{2} c v_{q} \eta_{q} \eta_{s} .
\end{aligned}
$$

After diagonalization, the new eigenstates are $\tilde{\sigma}, \tilde{\eta}$ and $\tilde{\eta}^{\prime}$. 
Their dependence on $\mu_{5}$ is shown on Fig.5.
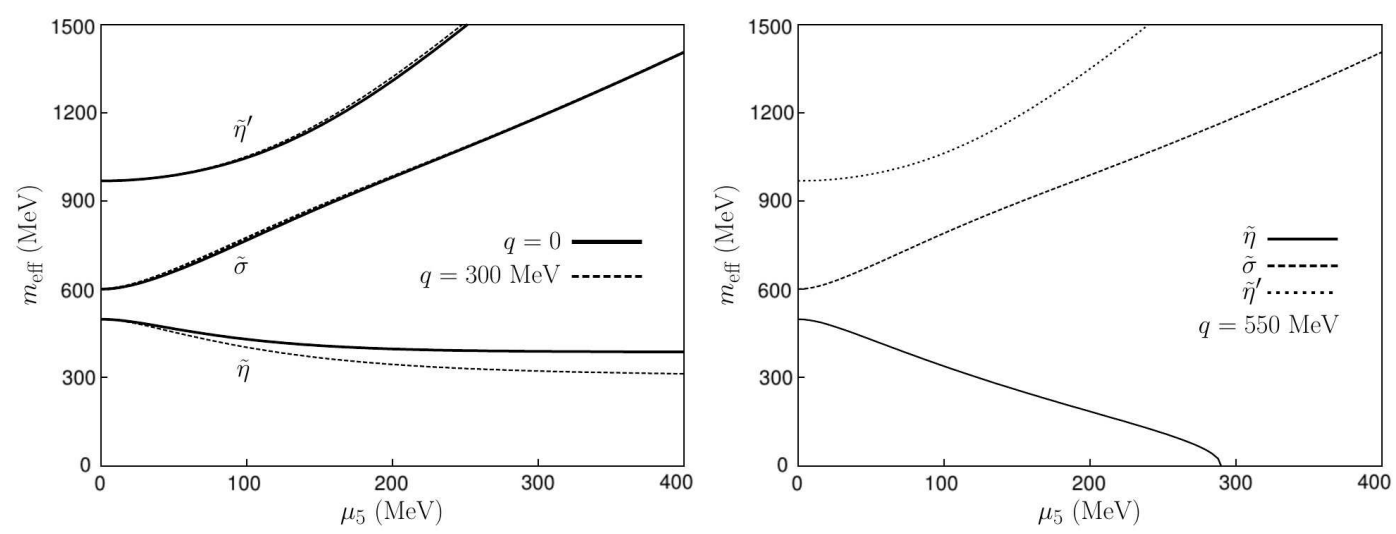

Figure 5. For high energies $k_{0},|\vec{k}|>k_{\tilde{\eta}}^{c}$ with $k_{\tilde{\eta}}^{c} \equiv \frac{m_{3}}{4 \mu_{5} m_{5}} \sqrt{m_{4}^{2} m_{5}^{2}-8 c^{2} v_{q}^{2}}$, in-medium $\tilde{\eta}$ goes tachyonic.

$\tilde{\eta}^{\prime}$ mass also shows an enhancement and a better treatment would require the inclusion of heavier degrees of freedom.

The widths are firstly computed at the rest frame of the decaying particle and secondly with a boosted particle as shown on the following plots.
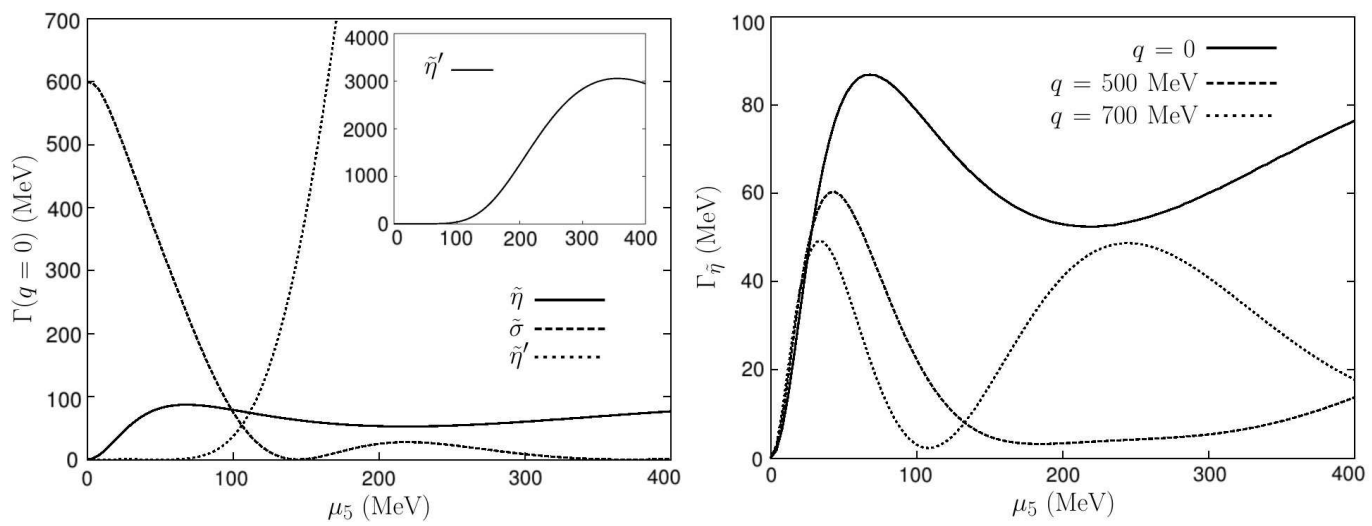

$\tilde{\eta}$ exhibits a smooth behaviour with $\left\langle\Gamma_{\tilde{\eta}}\right\rangle \sim 60 \mathrm{MeV}$ and the mean free path becomes $\sim 3 \mathrm{fm}$ $\lesssim L_{\text {fireball }} \sim 5 \div 10 \mathrm{fm}$. Thus its thermalization may be possible! Down to $\mu_{5} \sim 100 \mathrm{MeV}, \tilde{\sigma}$ width decreases and it becomes stable. The visible bumps in these two channels seem to reflect the tachyonic nature of the decaying $\tilde{\pi}$. $\tilde{\eta}^{\prime}$ width grows up to the $\mathrm{GeV}$ scale (violation of unitarity).

\section{Baryon and chiral chemical potentials in the NJL model}

Lattice simulations are efficient for various temperatures but suffer from serious problems when baryon chemical potential is sufficiently large. This is why different simplifications of QCD in the quark sector are usually employed in order to explore the baryon density influence of the QCD phase 
diagram [20-23]. In [24] we incorporated both a vector and an axial chemical potentials in the NambuJona-Lasinio (NJL) model with the purpose of unraveling the landscape of different stable phases of the theory. It turns out that the inclusion of $\mu_{5}$ changes radically the phase structure of the model and shows that $\mu$ is not a key player in ushering a thermodynamically stable phase where parity is violated in the NJL model, but $\mu_{5}$ is. It leads to a non-trivial dependence of the scalar condensate in the chirally broken phase (see Fig.6). Critical temperature with chiral chemical potential switched on
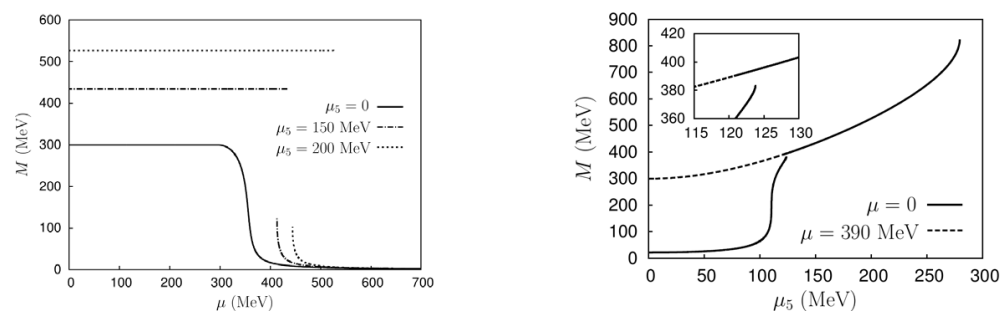

Figure 6. Evolution of the constituent quark mass $M$ [24]: left panel- depending on $\mu$ for different values of the axial chemical potential $\mu_{5}$; right panel- depending on $\mu_{5}$ for different values of the chemical potential $\mu$.

was computed on lattice [10]. The main result is that with increasing chiral chemical potential the dynamical mass and critical temperature raise up. For finite baryon densities the similar rise of the dynamical mass and critical point was established in [24], see Fig.6.

However in recent literature different controversial conclusions appeared last few years based on different models of NJL type. Among them an opposite decreasing behavior of quark condensate and dynamical mass with increasing temperatures was found in [25-27] in contrast to the lattice results[10]. Recently this controversy was thoroughly elucidated as an artefact of ultraviolet regularization misuse [28, 29]. Eventually the results of [24] fit well the QCD expectations supported by lattice simulations[10].

\section{Vector mesons in a medium with LPB}

LPB can be investigated in e.m. production of leptons and photons in hot/dense nuclear matter via heavy ion collisions.

- Thus e.m. interaction extends the axial charge by e.m. anomaly,

$$
Q_{5}^{q} \rightarrow \tilde{Q}_{5}=Q_{5}^{q}-T_{5}^{\mathrm{em}}, \quad T_{5}^{\mathrm{em}}=\frac{N_{c}}{8 \pi^{2}} \int_{\text {vol. }} d^{3} x \varepsilon_{j k l} \operatorname{Tr}\left(\hat{A}^{j} \partial^{k} \hat{A}^{l}\right)
$$

- Now chiral chemical potential $\mu_{5}$ is conjugated to (nearly) conserved $\tilde{Q}_{5}$.

- In the hadron phase QCD bosonization of total $\tilde{Q}_{5}$ can be realized [11] with the help of meson Lagrangian and the Vector Meson Dominance providing a good precision in coefficients.

$$
\begin{gathered}
\mathcal{L}_{\mathrm{int}}=\bar{q} \gamma_{\mu} \hat{V}^{\mu} q ; \quad \hat{V}_{\mu} \equiv-e A_{\mu} Q+\frac{1}{2} g_{\omega} \omega_{\mu} \mathbb{I}+\frac{1}{2} g_{\rho} \rho_{\mu}^{0} \tau_{3}, \\
\left(V_{\mu, a}\right) \equiv\left(A_{\mu}, \omega_{\mu}, \rho_{\mu}^{0}\right)
\end{gathered}
$$

where $Q=\frac{\tau_{3}}{2}+\frac{1}{6}, g_{\omega} \simeq g_{\rho} \equiv g \simeq 6$. 
In the parity-odd effective lagrangian for vector mesons the following term is generated

$$
\Delta \mathcal{L} \simeq \varepsilon^{\mu \nu \rho \sigma} \operatorname{Tr}\left[\zeta_{\mu} V_{v} V_{\rho \sigma}\right]
$$

with $\zeta_{\mu}=\zeta \delta_{\mu 0}$ for a spatially homogeneous and isotropic background and $\zeta \propto \mu_{5}$.

Two different cases of isospin structure for $\mu_{5}$ :

- Isosinglet pseudoscalar background $(T \gg \mu)$ [RHIC, LHC]

- Pion-like (isotriplet) background $(\mu \gg T)$ [FAIR, NICA]

Massive MCS electrodynamics for vector mesons is described by the following lagrangian

$$
\mathcal{L}_{M C S}=-\frac{1}{4} F^{\alpha \beta}(x) F_{\alpha \beta}(x)+\frac{1}{2} m^{2} A_{v}(x) A^{v}(x)+\frac{1}{2} \zeta_{\mu} A_{v}(x) \tilde{F}^{\mu v}(x)+\text { g.f. }
$$

In momentum space wave Eqs.

$$
\left[g^{\lambda v}\left(k^{2}-m^{2}\right)-k^{\lambda} k^{v}+i \varepsilon^{\lambda v \alpha \beta} \zeta_{\alpha} k_{\beta}\right] \mathbf{a}_{\lambda}(k)=0 ; \quad k^{\lambda} \mathbf{a}_{\lambda}(k)=0 .
$$

Energy spectrum for transversal polarizations is governed by

$$
\omega_{\mathbf{k}, \pm}=\sqrt{\mathbf{k}^{2}+m^{2} \pm \zeta|\mathbf{k}|},
$$

and for longitudinal polarization it remains intact,

$$
\omega_{\mathbf{k}, L}=\sqrt{\mathbf{k}^{2}+m^{2}}
$$

After diagonalization of mass matrix the mass spectrum is given by

$$
m_{V, \epsilon}^{2}=m_{V}^{2}-\epsilon \zeta|\vec{k}|
$$

where $\epsilon=0, \pm 1$ is the type of meson polarization. Thus $m_{V,+}^{2}<m_{V, L}^{2}<m_{V,-}^{2}$.

If $\hat{\zeta}$ is an isosinglet the photon itself happens to be unaffected by CS interaction. The position of the poles for \pm polarized vector mesons is moving with wave vector $|\vec{k}|$.

This splitting unambiguously signifies LPB. Can it be measured? Dilepton production in HIC from the decays $\rho, \omega \rightarrow e^{+} e^{-}$potentially contains sufficient information on LPB.

\subsection{Manifestation of LPB in heavy ion collisions in dilepton production}

$L, \pm$ contribution for vector mesons before acceptance corrections:

$$
\begin{aligned}
& \frac{d N_{e e}^{\epsilon}}{d M} \simeq c_{V} \frac{\alpha^{2} \Gamma_{V} m_{V}^{2}}{3 \pi^{2} g^{2} M^{2}}\left(\frac{M^{2}-n_{V}^{2} m_{\pi}^{2}}{m_{V}^{2}-n_{V}^{2} m_{\pi}^{2}}\right)^{3 / 2} \\
& \times \sum_{\epsilon} \int_{M}^{\infty} d k_{0} \frac{\sqrt{k_{0}^{2}-M^{2}}}{e^{k_{0} / T}-1} \frac{m_{V, \epsilon}^{4}}{\left(M^{2}-m_{V, \epsilon}^{2}\right)^{2}+m_{V, \epsilon}^{4} \frac{\Gamma_{V}^{2}}{m_{V}^{2}}}
\end{aligned}
$$

where $n_{V}=2,0 ;|\vec{k}|=\sqrt{k_{0}^{2}-M^{2}}$ and $M^{2}>n_{V}^{2} m_{\pi}^{2} \cdot c_{V}$ absorbs combinatorial factors different for $\rho$ and $\omega, \mu$, finite volume suppression.

Simulations of distorted spectral functions are implemented with PHENIX acceptance: $\left|y_{e e}\right|<$ $0.35,\left|\vec{p}_{t}^{e}\right|>200 \mathrm{MeV}$, gaussian $M_{e e}$ smearing (width=10 MeV) 

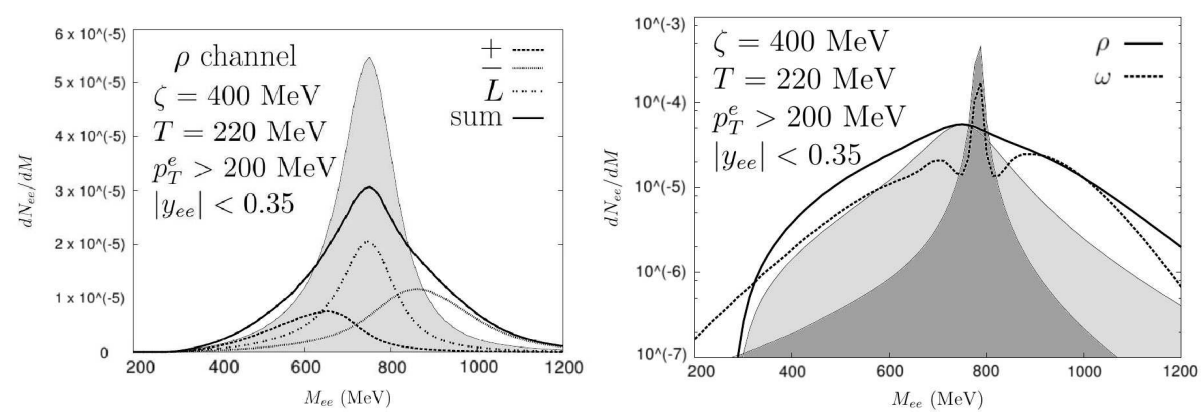

Figure 7. On the left: polarization splitting in $\rho$ spectral function for $\mathrm{LPB} \zeta=400 \mathrm{MeV}\left(\mu_{5}=290 \mathrm{MeV}\right)$ compared with $\zeta=0$ (shaded region). On the right: in-medium $\rho$ and $\omega$ channels (solid and dashed line) and their vacuum contributions (light and dark shaded regions) for $\zeta=400 \mathrm{MeV}$. In-medium $\rho$ is enhanced by a factor 1.8 due to $\pi \pi$ regeneration into $\rho$.

The PHENIX/STAR anomaly[30, 31] - an abnormal $e^{+} e^{-}$yield at low invariant mass is observed in central HIC. It may be explained partially by the local parity breaking shown in Fig.7.

\subsection{Polarization asymmetry: dilepton angular distributions}

In [32] it was suggested to detect a splitting of dilepton polarizations averaged over angular sectors. There are two convenient choices of this splitting.

Case A: the angle $\theta_{A}$ between the two outgoing leptons in the laboratory frame.
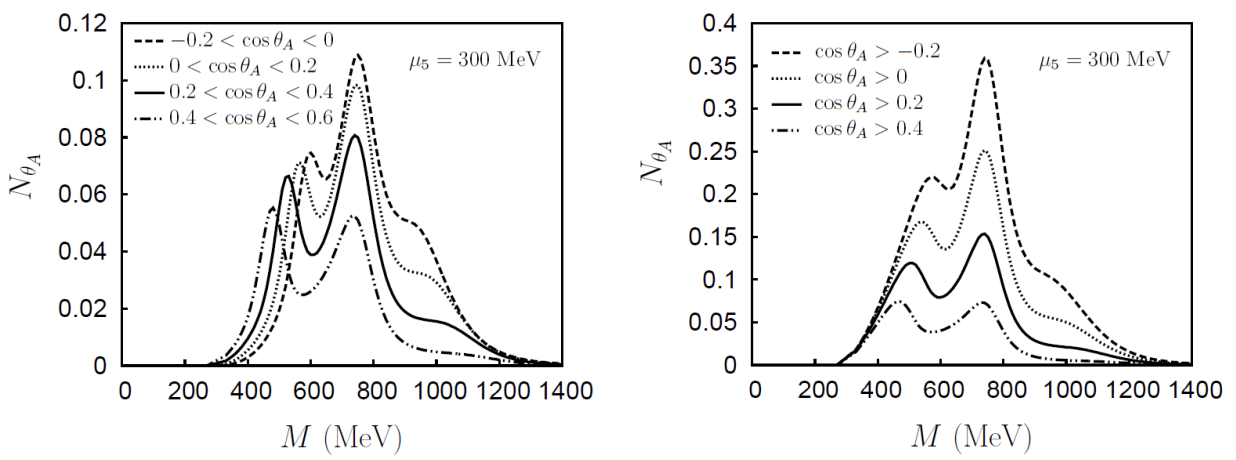

Figure 8. The $\rho$ spectral function is presented for fixed $\mu_{5}=300 \mathrm{MeV} \cdot \cos \theta_{A} \in$ $[-0.2,0],[0,0.2],[0.2,0.4],[0.4,0.6]$ and $[0.6,0.8]$ in the left panel, and $\cos \theta_{A} \geq-0.2,0,0.2,0.4$ in the right one. Results are corrected for PHENIX experimental acceptance.

Case B: the angle $\theta_{B}$ between one of the two outgoing leptons in the laboratory frame and the same lepton in the dilepton rest frame 

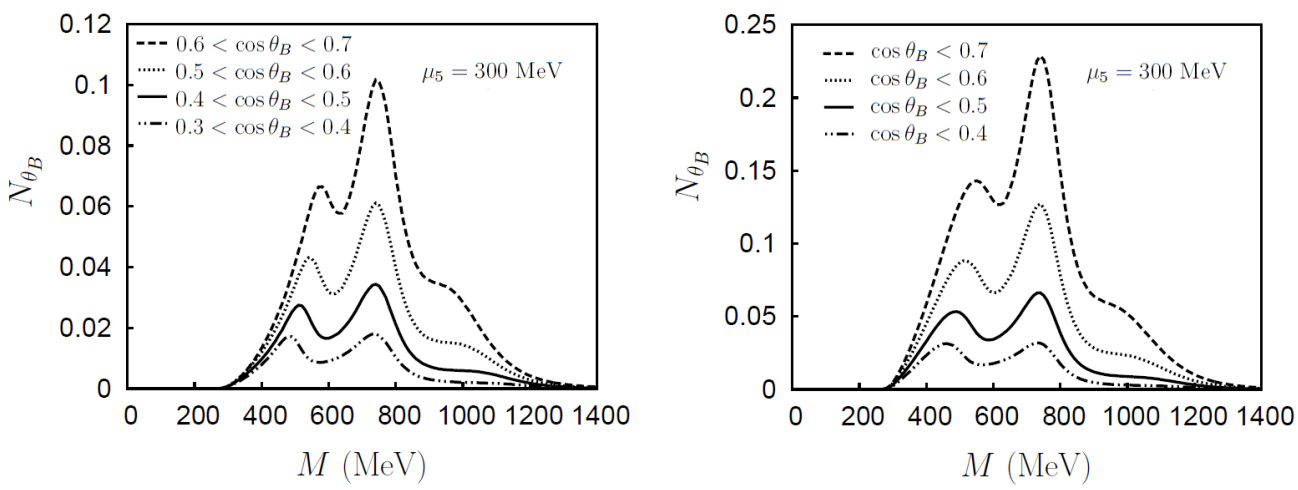

Figure 9. The $\rho$ spectral function is presented for fixed $\mu_{5}=300 \mathrm{MeV} \cdot \cos \theta_{B} \in[0.3,0.4],[0.4,0.5],[0.5,0.6]$ and $[0.6,0.7]$ in the left panel, and $\cos \theta_{B} \leq 0.4,0.5,0.6,0.7$ in the right one. Results are corrected for PHENIX experimental acceptance.

\section{Conclusions and Outlook}

In this report the situation when in an environment with chiral imbalance of quarks which formed as a result of heavy-ion collisions at high energies the possibility of the formation of phase with a Local spatial Parity Breaking (LPB) were considered.

We have established that:

1.To describe such a quark (hadron) matter topological charge fluctuations transmit their influence to hadron physics via axial(chiral) chemical potential which can be introduced to quantify the presence of chirality imbalance. In particular, in a hadron phase some of experimental observables are presented for detecting the signal of LPB. The asymmetry in production of longitudinally and transversely polarized states of $\rho$ and $\omega$ mesons for various values of the dilepton invariant mass can serve as a characteristic indication of the LPB in CERES, HADES, PHENIX, STAR, NA60 and ALICE experiments.

2.LPB enhances dynamical chiral symmetry breaking in QCD: chiral condensates, critical temperature of chiral symmetry restoration are increasing with chiral chemical potential.

3. Axial chemical potential triggers parity-odd condensation for large baryon chemical potential in first-order phase transition ("chiral catalysis").

4.LPB modifies dispersion laws for scalar and vector mesons: lightest "pseudoscalar" mesons tend to massless states in flight, vector meson polarizations split with different in-flight masses.

5.There are observables unambiguously indicating LPB (ALICE LHC?)

6.QCD-motivated models are considered from whose properties the phenomenon of LPB could be found. The results are compared with lattice calculations for the investigated phases.

\section{Acknowledgements}

A.A.and D.E. express their appreciation to the organizing Committee of the XIIth international conference Quark Confinement and Hadron Spectrum, for the excellent hospitality and organization of this conference. This work has been supported through grants FPA2013-46570, 2014-SGR-104 and Consolider CPAN. Funding was also partially provided by the Spanish MINECO under project MDM2014-0369 of ICCUB (Unidad de Excelencia 'Maria de Maeztu'). A.A. and V.A. were supported by 
Grant RFBR projects 16-02-00348 and also got a financial support of SPbSU, in particular, by projects 11.42.1049.2016, 11.42.1051.2016.

\section{References}

[1] A.A. Belavin, A.M. Polyakov, A.S. Schwartz and Y. S. Tyupkin, Phys. Lett. B 59, 85 (1975).

[2] C. G. Callan, Jr., R. F. Dashen and D. J. Gross, Phys. Lett. B 63, 334 (1976).

[3] G. 't Hooft, Phys. Rev. D 14, 3432 (1976) [Erratum-ibid. D 18,2199 (1978)].

[4] D.I. Dyakonov and V.Y. Petrov, Phys. Lett. B 147, 351 (1984); Sov. Phys. JETP 62, 204 (1985); ibid. 62, 431 (1985); Nucl. Phys. B 272, 457 (1986).

[5] T. Schaefer and E. V. Shuryak, Rev. Mod. Phys. 70, 323 (1998); [hep-ph/9610451]

[6] S. L. Adler, Phys. Rev. 177, 2426 (1969).

[7] J. S. Bell and R. Jackiw, Nuovo Cim. A 60, 47 (1969).

[8] D.E. Kharzeev, R.D. Pisarski, M.H.G. Tytgat, Phys. Rev. Lett. 81, 512 (1998); K. Buckley, T. Fugleberg, and A. Zhitnitsky, Phys. Rev. Lett. 84, 4814 (2000); D.E. Kharzeev, Phys. Lett. B 633, 260 (2006); D.E. Kharzeev, Ann. Phys. (NY) 325, 205 (2010). D.E. Kharzeev, A. Zhitnitsky, Nucl. Phys. A 797, 67 (2007). D.E. Kharzeev, L.D. McLerran, H.J. Warringa, Nucl. Phys. A 803, 227 (2008). K. Fukushima, D.E. Kharzeev, H.J. Warringa, Phys. Rev. D 78, 074033 (2008);, K. Fukushima, D.E. Kharzeev, H.J. Warringa, Nucl. Phys. A 836, 311 (2010).

[9] B. Wang, Y. L. Wang, Z. F. Cui and H. S. Zong, Phys. Rev. D 91, 034017 (2015); S. S. Xu, Z. F. Cui, B. Wang, Y. M. Shi, Y. C. Yang and H.S.Zong,Phys. Rev. D 91, 056003 (2015).

[10] V. V. Braguta, V. A. Goy, E. M. Ilgenfritz, A. Yu. Kotov, A. V. Molochkov, M. Muller-Preussker, and B. Petersson. JHEP, 06:094, 2015; V. V. Braguta and A. Y. Kotov, Phys. Rev. D 93, no. 10, 105025 (2016).

[11] A.A. Andrianov, V.A. Andrianov, D. Espriu, X. Planells, Phys. Lett. B 710,230 (2012); Theor. Math. Phys. 170, 17 (2012) [Teor. Mat. Fiz. 170, 22 (2012)]; Phys. Rev. D 90, 034024 (2014).

[12] G. 't Hooft, hep-th/0010225; T. Schaefer and E. V. Shuryak, Rev. Mod. Phys. 70, 323 (1998).

[13] T. D. Lee and G. C. Wick Phys. Rev. D 9, 2291 (1974).

[14] A.Vilenkin, Phys.Rev. D 22, 3080 (1980).

[15] A.A.Anselm, Phys.Lett. B 217,169 (1989); A.A.Anselm, M.G.Ryskin, A.G.Shuvaev, Surv. in HEP 11, 27 (1997).

[16] A.A. Andrianov, D. Espriu, Phys. Lett. B 663,450 (2008); A.A. Andrianov, V.A. Andrianov, D. Espriu, Phys. Lett. B 678,416 (2009).

[17] F. R. Klinkhamer and N. S. Manton, Phys. Rev. D 30, 2212 (1984); V. A. Kuzmin, V. A. Rubakov, and M. E. Shaposhnikov, Phys. Lett. B 155, 36 (1985); L. McLerran, E. Mottola, and M. E. Shaposhnikov, Phys.Rev. D 43, 2027 (1991); G. D. Moore and K. Rummukainen, Phys. Rev. D 61, 105008 (2000); E. Shuryak and I. Zahed, Phys. Rev. D 67, 014006 (2003).

[18] R. Rapp and J. Wambach, in: Advances in Nuclear Physics (Adv. Phys. Part. Nucl. (APPN), Vol. 25, J. W. Negele and E. Vogt, eds.), Kluwer, New York (2002), pp. 1-205; H. van Hees and R. Rapp, Nucl. Phys. A 806, 339 (2008).

[19] A. A. Andrianov, D. Espriu \& X. Planells, Eur. Phys. J. C 73:2294 (2013).

[20] Y. Nambu and G. Jona-Lasinio, Phys. Rev. 122,345,(1961); Phys. Rev.124,246,(1961).

[21] U. Vogl, W. Weise, Prog. Part. Nucl. Phys. 27,195(1991).

[22] S. P. Klevansky, Rev. Mod. Phys. 64, 649, (1992); T. Hatsuda and T. Kunihiro, Phys. Rept. 247, 221 (1994); M. Buballa, Phys. Rept. 407 , 205, (2005).

[23] D. Ebert, K. G. Klimenko, Eur. Phys. J C 46,771, (2006). 
[24] A. A. Andrianov, D. Espriu, and X. Planells, Eur. Phys. J. C 74, 2776 (2014).

[25] M.N.Chernodub and A.S.Nedelin Phys. Rev. D 83, 105008 (2011).

[26] M.R.Ruggieri, Phys.Rev. D 84, 014011 (2011).

[27] Lang Yu, Hao Liu, and Mei Huang Phys. Rev. D 94, 014026 (2016).

[28] R.L.S.Farias, D.C.Duarte, G.Krein and R.O.Ramos, Phys. Rev. D 94,074011 (2016).

[29] M.Ruggieri and G.X.Peng, J. Phys. G: Nucl. Part. Phys. 43, 125101 (2016).

[30] A. Adare et al. [PHENIX Collaboration], Phys. Rev. C 81, 034911 (2010), arXiv:0912.0244 [nucl-ex].

[31] L. Adamczyk et al. [STAR Collaboration], Phys. Rev. Lett. 113, no. 2, 022301 (2014) Addendum: [Phys. Rev. Lett. 113, no. 4, 049903 (2014)] arXiv:1312.7397 [hep-ex].

[32] A.A. Andrianov, V.A. Andrianov, D. Espriu, X. Planells, Phys. Rev. D 90, 034024 (2014); A.A. Andrianov, V.A. Andrianov, D. Espriu, Phys. Part. Nucl. 41, 896 (2010); Acta Phys.Polon.Supp. 9, 515, (2016). 